\title{
ON CERTAIN HOMOLOGICAL FINITENESS CONDITIONS
}

\author{
DIMITRIOS BALLAS
}

\begin{abstract}
In this paper, we show that the injective dimension of all projective modules over a countable ring is bounded by the self-injective dimension of the ring. We also examine the extent to which the flat length of all injective modules is bounded by the flat length of an injective cogenerator. To that end, we study the relation between these finiteness conditions on the ring and certain properties of the (strict) Mittag-Leffler modules. We also examine the relation between the self-injective dimension of the integral group ring of a group and Ikenaga's generalized (co-)homological dimension.
\end{abstract}

\section{INTRODUCTION}

The universal property of inverse limits of left $R$-modules may be reformulated as the assertion that the covariant Hom-functor $\operatorname{Hom}_{R}\left(A,{ }_{-}\right)$commutes with such limits for any left $R$-module $A$. Similarly, the universal property of direct limits of left $R$-modules may be reformulated as the assertion that the contravariant Hom-functor $\operatorname{Hom}_{R}(-, A)$ maps such limits to the corresponding inverse limits of Hom-groups for any left $R$-module $A$.

On the other hand, the requirement that the covariant $\operatorname{Hom}_{-}$functor $\operatorname{Hom}_{R}\left(A,_{-}\right)$commutes with direct limits imposes certain restrictions on $A$ : If $\left(B_{i}\right)_{i}$ is a direct system of left $R$ modules, the canonical maps of the $B_{i}$ 's to the direct limit $\lim _{\longrightarrow} B_{i}$ induce an additive map

$$
\lim _{\longrightarrow} \operatorname{Hom}_{R}\left(A, B_{i}\right) \longrightarrow \operatorname{Hom}_{R}\left(A, \lim _{\longrightarrow} B_{i}\right)
$$

It turns out that the above map is bijective for any direct system $\left(B_{i}\right)_{i}$ of left $R$-modules if and only if the left $R$-module $A$ is finitely presented. More generally, the canonical maps of the $B_{i}$ 's to the direct $\operatorname{limit}_{\longrightarrow} \lim _{i} B_{i}$ induce an additive map

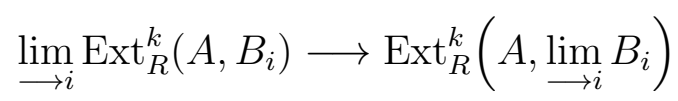

for any left $R$-module $A$ and any $k \in \mathbb{N}$. If $n$ is a non-negative integer, then one can show that the latter map is bijective for any direct system $\left(B_{i}\right)_{i}$ of left $R$-modules and any $k \leq n$ if and only if the left $R$-module $A$ is of type $\mathrm{FP}_{n+1}$, i.e. if and only if there exists a projective resolution $P_{*} \longrightarrow A \longrightarrow 0$, with $P_{i}$ finitely generated (and projective) for all $i \leq n+1$.

In general, one may look for conditions under which the vanishing of the groups $\operatorname{Ext}_{R}^{k}\left(A, B_{i}\right)$ implies that $\operatorname{Ext}_{R}^{k}\left(A, \lim _{\longrightarrow} B_{i}\right)=0$. In particular, if $B$ is a left $R$-module, one may ask whether the vanishing of the functor $\operatorname{Ext}_{R}^{k}(-, B)$ implies the vanishing of the functors $\operatorname{Ext}_{R}^{k}\left(-, B^{(\Lambda)}\right)$ for any set $\Lambda$. In other words, one may look for a relation between the injective dimension of $B$ and that of direct sums of copies of it. In the special case where $B=R$ is the left regular module, one then looks for a relation between the self-injective dimension of $R$ and the injective dimension of projective left $R$-modules. It is known that we have an inequality

Research supported by a GSRT/Greece excellence grant, cofunded by the ESF/EU and National Resources. 
$\operatorname{id}_{R} P \leq \operatorname{id}_{R} R$ for any projective left $R$-module if the ring $R$ is left Noetherian or else if $R$ is left perfect and right coherent. In that direction, we shall prove the following result:

Theorem. If $R$ is a countable ring, then we have an inequality $i d_{R} P \leq i d_{R} R$ for any projective left $R$-module $P$.

If $G$ is a countable group, then the finiteness of the self-injective dimension of the integral group ring $\mathbb{Z} G$ has important geometric consequences, which are analyzed in [16]. On the other hand, the self-injective dimension of $\mathbb{Z} G$ is closely related to the generalized (co-)homological dimension of $G$, which was defined by Ikenaga in [12].

Coming back to the general case of a ring $R$, we note that statements analogous to the above are valid for the tensor product functors, which are well-known to commute with direct limits: If $\left(B_{i}\right)_{i}$ is any direct system of left $R$-modules, then the canonical maps of the $B_{i}$ 's to the direct limit $\lim _{\longrightarrow} B_{i}$ induce an isomorphism

$$
\lim _{\longrightarrow}\left(A \otimes_{R} B_{i}\right) \longrightarrow A \otimes_{R}\left(\lim _{\longrightarrow} B_{i}\right)
$$

for any right $R$-module $A$. In particular, the tensor product functor $A \otimes_{R}$ - commutes with direct sums. On the other hand, the requirement that the tensor product functor $A \otimes_{R}-$ commutes with inverse limits and, in particular, with direct products imposes certain restrictions on $A$. More precisely, for any family of left $R$-modules $\left(B_{i}\right)_{i}$ the projection maps of the direct product $\prod_{i} B_{i}$ to the $B_{i}$ 's induce an additive map

$$
A \otimes_{R}\left(\prod_{i} B_{i}\right) \longrightarrow \prod_{i}\left(A \otimes_{R} B_{i}\right)
$$

for any right $R$-module $A$. The additive map above is surjective for any family $\left(B_{i}\right)_{i}$ of left $R$ modules if and only if the right $R$-module $A$ is finitely generated. The injectivity of that map is more subtle and leads to the notion of Mittag-Leffler modules, which were introduced by Raynaud and Gruson in [15]. More generally, one may consider for any non-negative integer $k$ the additive map

$$
\operatorname{Tor}_{k}^{R}\left(A, \prod_{i} B_{i}\right) \longrightarrow \prod_{i} \operatorname{Tor}_{k}^{R}\left(A, B_{i}\right),
$$

which is induced by the projection maps of the direct product $\prod_{i} B_{i}$ to the $B_{i}$ 's, and look for conditions under which the vanishing of the groups $\operatorname{Tor}_{k}^{R}\left(A, B_{i}\right)$ implies that $\operatorname{Tor}_{k}^{R}\left(A, \prod_{i} B_{i}\right)=$ 0 . In particular, if $B$ is a left $R$-module, one may ask whether the vanishing of the functor $\operatorname{Tor}_{k}^{R}\left({ }_{-}, B\right)$ implies the vanishing of the functors $\operatorname{Tor}_{R}^{k}\left({ }_{-}, B^{\Lambda}\right)$ for any set $\Lambda$. In other words, one may look for a relation between the flat dimension of $B$ and that of direct products of copies of it. In the special case where $B=\operatorname{Hom}(R, \mathbb{Q} / \mathbb{Z})$ is the Pontryagin dual of the regular right $R$-module $R$, one then looks for a relation between the flat dimension of $\operatorname{Hom}(R, \mathbb{Q} / \mathbb{Z})$ and the flat dimension of injective left $R$-modules. In that direction, we shall prove the following result:

Theorem. Let $R$ be a right $\aleph_{0}$-Noetherian ring and assume that all projective right $R$-modules have injective dimension $\leq 1$. Then, we have an inequality $f d_{R} I \leq f d_{R} \operatorname{Hom}(R, \mathbb{Q} / \mathbb{Z})$ for any injective left $R$-module $I$.

We note that the class of right $\aleph_{0}$-Noetherian rings over which projective right modules have injective dimension $\leq 1$ includes the quasi-Frobenius rings and the integral group rings of finite groups. 
The proof of both of the above results uses the properties of the (strict) Mittag-Leffler modules that were defined by Raynaud and Gruson in [15]. We recall this notion and detail the results that will be needed in sections 1 and 2 . Then, we relate the injective dimension of the left regular module to the injective dimension of projective left $R$-modules (in section 3 ) and the flat dimension of an injective cogenerator of the category of left $R$-modules to the flat dimension of injective left $R$-modules (in section 5 ). In section 4 , we examine the relation between the injective dimension of projective modules over the integral group ring $\mathbb{Z} G$ of a group $G$ and Ikenaga's generalized homological dimension hd $G$.

Notations and terminology. For any two abelian groups $M, N$ we denote by $\operatorname{Hom}(M, N)$ the group $\operatorname{Hom}_{\mathbb{Z}}(M, N)$ of all additive maps from $M$ to $N$. If $R$ is a ring and $\left(B_{i}\right)_{i}$ is a family of left $R$-modules, then for any left $R$-module $A$ we identify the abelian group $\operatorname{Hom}_{R}\left(A, \prod_{i} B_{i}\right)$ with the direct product $\prod_{i} \operatorname{Hom}_{R}\left(A, B_{i}\right)$. In this way, a family $\left(f_{i}\right)_{i} \in \prod_{i} \operatorname{Hom}_{R}\left(A, B_{i}\right)$ is identified with the $R$-linear map $f: A \longrightarrow \prod_{i} B_{i}$, which is given by $a \mapsto\left(f_{i}(a)\right)_{i}, a \in A$. Finally, we denote by $D$ the Pontryagin duality functors from the category of left (resp. right) $R$-modules to the category of right (resp. left) $R$-modules, which are defined by $M \mapsto \operatorname{Hom}(M, \mathbb{Q} / \mathbb{Z})$.

\section{Mittag-Leffler modules}

In this section, we review the notion of a Mittag-Leffler module and record some properties of these modules that will be used in the sequel.

First of all, we recall the Mittag-Leffler condition for an inverse system, introduced by Grothendieck in $[11, \S 13.1 .2]$. An inverse system of abelian groups $\left(A_{i}\right)_{i}$ with structural maps $\sigma_{i j}: A_{j} \longrightarrow A_{i}, i \leq j$, is said to satisfy the Mittag-Leffler condition if for any index $i$ there exists an index $j=j(i) \geq i$, such that

$$
\operatorname{im}\left(A_{j} \stackrel{\sigma_{i j}}{\longrightarrow} A_{i}\right)=\operatorname{im}\left(A_{k} \stackrel{\sigma_{i k}}{\longrightarrow} A_{i}\right)
$$

for all $k \geq j$. Assuming that the inverse system $\left(A_{i}\right)_{i}$ satisfies the Mittag-Leffler condition, we refer to the subgroup $A_{i}^{\prime}=\operatorname{im}\left(A_{j} \stackrel{\sigma_{i j}}{\longrightarrow} A_{i}\right)$, where $j=j(i)$ as above, as the stable image.

The notion of a Mittag-Leffler module was introduced by Raynaud and Gruson in [15] and has been studied subsequently by many authors; the reader may consult the detailed exposition [1]. This notion describes a property that a module may enjoy, as far as the representation of it as a direct limit of finitely presented modules is concerned. More precisely, let $M$ be a right $R$-module and express $M$ as the direct limit of a direct system $\left(M_{i}\right)_{i}$ of finitely presented right $R$-modules. Then, $M$ is a Mittag-Leffler module if the inverse system of abelian groups $\left(\operatorname{Hom}_{R}\left(M_{i}, N\right)\right)_{i}$, whose structural maps are induced by the structural maps of the direct system $\left(M_{i}\right)_{i}$, satisfies the Mittag-Leffler condition for any right $R$-module $N$. This definition does not depend upon the particular representation of $M$ as the direct limit of a direct system of finitely presented right $R$-modules and may be equivalently formulated by means of the injectivity of certain natural maps. For any family of left $R$-modules $\left(N_{i}\right)_{i}$ we may consider the additive map

$$
\varphi_{M}: M \otimes_{R}\left(\prod_{i} N_{i}\right) \longrightarrow \prod_{i}\left(M \otimes_{R} N_{i}\right)
$$

which is given by letting $m \otimes\left(n_{i}\right)_{i} \mapsto\left(m \otimes n_{i}\right)_{i}$ for any $m \in M$ and $\left(n_{i}\right)_{i} \in \prod_{i} N_{i}$. We note that $\varphi_{M}=\left(1 \otimes p_{i}\right)_{i}$, where $p_{i}$ denotes the $i$-th coordinate projection map of the direct product onto $N_{i}$ for any index $i$. Then, as shown in [15], the right $R$-module $M$ is Mittag-Leffler if and only if $\varphi_{M}$ is injective for any family of left $R$-modules $\left(N_{i}\right)_{i}$. 
In the sequel, we shall need a relative version of this notion. If $\mathfrak{X}$ is a class of left $R$-modules, then we say that a right $R$-module $M$ is Mittag-Leffler with respect to $\mathfrak{X}$ if the additive map (1) is injective for any family of right $R$-modules $\left(N_{i}\right)_{i}$ that are contained in $\mathfrak{X}$. We denote by $\operatorname{ML}(\mathfrak{X})$ the class consisting of those right $R$-modules which are Mittag-Leffler over $\mathfrak{X}$. In the special case where $\mathfrak{X}=\{N\}$ is a singleton consisting of a left $R$-module $N$, we denote $\operatorname{ML}(\mathfrak{X})$ simply by $\operatorname{ML}(N)$.

Remarks 1.1. (i) If $\mathfrak{X}, \mathfrak{Y}$ are two classes of left $R$-modules and $\mathfrak{X} \subseteq \mathfrak{Y}$, then we obviously have an inclusion $\operatorname{ML}(\mathfrak{Y}) \subseteq \operatorname{ML}(\mathfrak{X})$.

(ii) Let $\mathfrak{X}$ be a class of left $R$-modules and consider the class $\overline{\mathfrak{X}}$ consisting of those left $R$-modules that may be embedded as direct summands in suitable direct products of modules contained in $\mathfrak{X}$. Then, it is easily seen that $\operatorname{ML}(\mathfrak{X})=\operatorname{ML}(\overline{\mathfrak{X}})$.

(iii) In particular, it follows from (i) and (ii) above that whenever $\mathfrak{X}, \mathfrak{Y}$ are two classes of left $R$-modules, such that $\mathfrak{X} \subseteq \mathfrak{Y}$ and any module of $\mathfrak{Y}$ may be embedded as a direct summand in a suitable direct product of modules contained in $\mathfrak{X}$, then we have an equality $\operatorname{ML}(\mathfrak{X})=\operatorname{ML}(\mathfrak{Y})$.

(iv) Let $J$ be an injective cogenerator of the category of left $R$-modules. Since any injective left $R$-module is a direct summand of a suitable product of copies of $J$, it follows from (iii) above that $\operatorname{ML}(J)=\operatorname{ML}(\mathfrak{I})$, where $\mathfrak{I}=\mathfrak{I}(R)$ is the class of injective left $R$-modules.

(v) Let $\mathfrak{X}$ be a class of left $R$-modules and consider a family $\left(M_{\lambda}\right)_{\lambda}$ of right $R$-modules and the direct sum $M=\bigoplus_{\lambda} M_{\lambda}$. Then, $M \in \mathrm{ML}(\mathfrak{X})$ if and only if $M_{\lambda} \in \operatorname{ML}(\mathfrak{X})$ for any $\lambda$. Indeed, if $\left(N_{i}\right)_{i}$ is any family of left $R$-modules in $\mathfrak{X}$, then it is easily seen that the additive map $\varphi_{M}$ is injective if and only if the corresponding maps $\varphi_{M_{\lambda}}$ are injective for all $\lambda$. Since the regular right $R$-module $R$ is obviously contained in $\operatorname{ML}(\mathfrak{X})$, we conclude that $\operatorname{ML}(\mathfrak{X})$ contains all projective right $R$-modules.

(vi) Let $\mathfrak{X}$ be a class of left $R$-modules and fix a non-negative integer $n$. For any right $R$ module $M$ we consider a projective resolution and denote by $K_{n}$ the corresponding $n$-th syzygy module. Of course, $K_{n}$ depends upon the choice of the particular resolution. Nevertheless, Schanuel's lemma implies that for any two $n$-th syzygy modules $K_{n}$ and $K_{n}^{\prime}$ of $M$ there are projective modules $P$ and $P^{\prime}$, such that $K_{n} \oplus P \simeq K_{n}^{\prime} \oplus P^{\prime}$. Since the class $\operatorname{ML}(\mathfrak{X})$ contains all projective right $R$-modules and is closed under direct sums and direct summands (in view of (v) above), we conclude that the right $R$-module $K_{n}$ is Mittag-Leffler with respect to $\mathfrak{X}$ if and only if this is the case for the right $R$-module $K_{n}^{\prime}$. In that case, we say that the $n$-th syzygy module of $M$ is Mittag-Leffler with respect to $\mathfrak{X}$.

The natural transformation $\varphi$ may be derived as follows: Having fixed a family $\left(N_{i}\right)_{i}$ of left $R$-modules, we consider for any right $R$-module $M$ a projective resolution $P_{*} \longrightarrow M \longrightarrow 0$ and the chain map

$$
\varphi_{P_{*}}: P_{*} \otimes_{R}\left(\prod_{i} N_{i}\right) \longrightarrow \prod_{i}\left(P_{*} \otimes_{R} N_{i}\right)
$$

By applying homology, we obtain additive maps

$$
\varphi_{M}^{(n)}: \operatorname{Tor}_{n}^{R}\left(M, \prod_{i} N_{i}\right) \longrightarrow \prod_{i} \operatorname{Tor}_{n}^{R}\left(M, N_{i}\right)
$$

$n \geq 0$, which do not depend upon the particular choice of the projective resolution of $M$. We note that $\varphi_{M}^{(n)}$ is the additive map whose $i$-th coordinate is that induced by applying the functor $\operatorname{Tor}_{n}^{R}\left(M,{ }_{-}\right)$to the $i$-th coordinate projection map $p_{i}$ of the direct product onto $N_{i}$ for any index $i$. It is clear that $\varphi_{M}^{(0)}$ can be identified with the map $\varphi_{M}$ studied before. Moreover, 
the $\varphi_{M}^{(n)}$ 's are natural in $M$ and commute with the connecting homomorphisms which are associated with any short exact sequence of left $R$-modules

$$
0 \longrightarrow M^{\prime} \longrightarrow M \longrightarrow M^{\prime \prime} \longrightarrow 0 \text {. }
$$

Proposition 1.2. Let $M$ be a right $R$-module and consider a class $\mathfrak{X}$ of left $R$-modules. Then, the following conditions are equivalent for a non-negative integer $n$ :

(i) The natural map

$$
\varphi_{M}^{(n)}: \operatorname{Tor}_{n}^{R}\left(M, \prod_{i} N_{i}\right) \longrightarrow \prod_{i} \operatorname{Tor}_{n}^{R}\left(M, N_{i}\right)
$$

is injective for any family $\left(N_{i}\right)_{i}$ of left $R$-modules contained in $\mathfrak{X}$.

(ii) The $n$-th syzygy module of $M$ is Mittag-Leffler with respect to $\mathfrak{X}$.

Proof. We proceed as in the proof of [6, Proposition 1.5]. Let $P_{*} \longrightarrow M \longrightarrow 0$ be a projective resolution of $M$ and consider the corresponding $n$-th syzygy module $K_{n}$. Then, $K_{n}$ fits into an exact sequence

$$
0 \longrightarrow K_{n} \longrightarrow P_{n-1} \longrightarrow \cdots \longrightarrow P_{1} \longrightarrow P_{0} \longrightarrow M \longrightarrow 0 .
$$

By applying dimension shifting, we obtain for any family $\left(N_{i}\right)_{i}$ of left $R$-modules exact sequences

$$
0 \longrightarrow \operatorname{Tor}_{n}^{R}\left(M, \prod_{i} N_{i}\right) \stackrel{a_{n}}{\longrightarrow} K_{n} \otimes_{R}\left(\prod_{i} N_{i}\right) \longrightarrow P_{n-1} \otimes_{R}\left(\prod_{i} N_{i}\right)
$$

and

$$
0 \longrightarrow \prod_{i} \operatorname{Tor}_{n}^{R}\left(M, N_{i}\right) \stackrel{b_{n}}{\longrightarrow} \prod_{i}\left(K_{n} \otimes_{R} N_{i}\right) \longrightarrow \prod_{i}\left(P_{n-1} \otimes_{R} N_{i}\right) .
$$

Since the additive maps $a_{n}, b_{n}$ are defined by composing connecting homomorphisms and $\phi^{(*)}$ commutes with connecting homomorphisms, we obtain a commutative diagram of abelian groups with exact rows

$$
\begin{aligned}
& 0 \longrightarrow \operatorname{Tor}_{n}^{R}\left(M, \prod_{\varphi_{M}^{(n)} \downarrow} N_{i}\right) \stackrel{a_{n}}{\longrightarrow} \begin{array}{c}
K_{n} \otimes_{R}\left(\prod_{i} N_{i}\right) \\
\downarrow
\end{array} \quad \begin{array}{c}
P_{n-1}^{(0)} \\
\otimes_{K_{n}}
\end{array} \otimes_{R}\left(\prod_{i} N_{i}\right) \\
& 0 \longrightarrow \prod_{i} \operatorname{Tor}_{n}^{R}\left(M, N_{i}\right) \stackrel{b_{n}}{\longrightarrow} \prod_{i}\left(K_{n} \otimes_{R} N_{i}\right) \longrightarrow \prod_{i}\left(P_{n-1} \otimes_{R} N_{i}\right)
\end{aligned}
$$

In view of Remark 1.1(v), the projective right $R$-module $P_{n-1}$ is Mittag-Leffler with respect to $\mathfrak{X}$ and hence the map $\varphi_{P_{n-1}}^{(0)}=\varphi_{P_{n-1}}$ is injective. Then, a diagram chase shows that $\varphi_{M}^{(n)}$ is injective if and only if this is the case for $\varphi_{K_{n}}^{(0)}=\varphi_{K_{n}}$, proving the equivalence between conditions (i) and (ii) in the statement.

\section{Strict Mittag-Leffler MOdules}

We shall now consider the notion of a strict Mittag-Leffler module and describe the relation between these modules and the Mittag-Leffler modules of the previous section.

We begin by defining the strict Mittag-Leffler condition for an inverse system of abelian groups: Let $\left(A_{i}\right)_{i}$ be an inverse system of abelian groups with structural maps $\sigma_{i j}: A_{j} \longrightarrow A_{i}$, $i \leq j$, and consider the inverse $\operatorname{limit} A=\lim _{\leftarrow} A_{i}$, which is endowed with canonical maps $s_{i}: A \longrightarrow A_{i}$ for any index $i$. The inverse system $\left(A_{i}\right)_{i}$ is said to satisfy the strict MittagLeffler condition if for any index $i$ there exists an index $j=j(i) \geq i$, such that

$$
\operatorname{im}\left(A_{j} \stackrel{\sigma_{i j}}{\longrightarrow} A_{i}\right)=\operatorname{im}\left(A \stackrel{s_{i}}{\longrightarrow} A_{i}\right) .
$$


The strict Mittag-Leffler condition for an inverse system is stronger that the Mittag-Leffler condition. In fact, it is easily seen that the inverse system $\left(A_{i}\right)_{i}$ satisfies the strict MittagLeffler condition if and only if it satisfies the Mittag-Leffler condition and the stable image $A_{i}^{\prime}$ coincides with the image of the canonical map $s_{i}: A \longrightarrow A_{i}$ for any index $i$. In the special case where the index set is countable, the strict Mittag-Leffler condition is actually equivalent to the Mittag-Leffler condition. This is implicit in [11, §13.2.2]; see also [5, Lemma 1.1].

The notion of a strict Mittag-Leffler module was introduced by Raynaud and Gruson in [15]. More precisely, we consider a right $R$-module $M$ and express it as the direct limit of a direct system $\left(M_{i}\right)_{i}$ of finitely presented right $R$-modules. Then, $M$ is a strict Mittag-Leffler module if the inverse system of abelian groups $\left(\operatorname{Hom}_{R}\left(M_{i}, N\right)\right)_{i}$, whose structural maps are induced by the structural maps of the direct system $\left(M_{i}\right)_{i}$, satisfies the strict Mittag-Leffler condition for any right $R$-module $N$. This definition does not depend upon the particular representation of $M$ as the direct limit of a direct system of finitely presented right $R$-modules and may be equivalently formulated by means of the injectivity of certain natural maps. If $N$ is a right $R$-module and $\Delta$ an abelian group, then the abelian group $\operatorname{Hom}(N, \Delta)$ of all additive maps from $N$ to $\Delta$ can be endowed with the structure of a left $R$-module, by using the right $R$-module structure of $N$. If $M$ is another right $R$-module, then we may consider the tensor product $M \otimes_{R} \operatorname{Hom}(N, \Delta)$ and define the map

$$
\Phi_{M}: M \otimes_{R} \operatorname{Hom}(N, \Delta) \longrightarrow \operatorname{Hom}\left(\operatorname{Hom}_{R}(M, N), \Delta\right),
$$

by letting $\Phi_{M}(m \otimes f)$ be the operator given by $g \mapsto f(g(m)), g \in \operatorname{Hom}_{R}(M, N)$, for all $m \in M$ and $f \in \operatorname{Hom}(N, \Delta)$. Then, as shown in [1, Theorem 8.11], the following two conditions are equivalent for a right $R$-module $M$ :

(i) If $M$ is expressed as the direct limit of a direct system $\left(M_{i}\right)_{i}$ of finitely presented right $R$-modules, then the inverse system of abelian groups $\left(\operatorname{Hom}_{R}\left(M_{i}, N\right)\right)_{i}$ satisfies the strict Mittag-Leffler condition.

(ii) The additive map (2) is injective for any divisible abelian group $\Delta$.

Having fixed the right $R$-module $N$, we say that a right $R$-module $M$ is strict Mittag-Leffler over $N$ if the equivalent conditions (i) and (ii) above are satisfied. We denote by $\operatorname{SML}(N)$ the class consisting of those right $R$-modules which are Mittag-Leffler over $N$.

Proposition 2.1. If $M, N$ are two right $R$-modules and $M$ is strict Mittag-Leffler over $N$, then $M$ is Mittag-Leffler with respect to the left $R$-module $D N=H o m(N, \mathbb{Q} / \mathbb{Z})$. In other words, we have an inclusion $\operatorname{SML}(N) \subseteq \operatorname{ML}(D N)$ for any right $R$-module $N$.

Proof. Our assumption on $M$ implies that the additive map (2) is injective for any divisible abelian group $\Delta$. Letting $T=\mathbb{Q} / \mathbb{Z}$, we have to show that for any set $I$ the additive map

$$
\varphi_{M}: M \otimes_{R} \operatorname{Hom}(N, T)^{I} \longrightarrow\left(M \otimes_{R} \operatorname{Hom}(N, T)\right)^{I}
$$

is injective. To that end, we consider the direct product $T^{I}$ and note that the coordinate projection maps $p_{i}: T^{I} \longrightarrow T, i \in I$, induce an isomorphism of left $R$-modules

$$
\left(p_{i *}\right)_{i}: \operatorname{Hom}\left(N, T^{I}\right) \longrightarrow \operatorname{Hom}(N, T)^{I} .
$$

Since the composition

$$
M \otimes_{R} \operatorname{Hom}\left(N, T^{I}\right) \stackrel{1 \otimes\left(p_{i *}\right)_{i}}{\longrightarrow} M \otimes_{R} \operatorname{Hom}(N, T)^{I} \stackrel{\varphi_{M}}{\longrightarrow}\left(M \otimes_{R} \operatorname{Hom}(N, T)\right)^{I}
$$

is easily seen to coincide with the additive map

$$
\left(1 \otimes p_{i *}\right)_{i}: M \otimes_{R} \operatorname{Hom}\left(N, T^{I}\right) \longrightarrow\left(M \otimes_{R} \operatorname{Hom}(N, T)\right)^{I},
$$


we are reduced to showing that (3) is injective. We now invoke the naturality of $\Phi$ with respect to the divisible abelian group and conclude that the following diagram is commutative

$$
\begin{array}{ccc}
M \otimes_{R} \operatorname{Hom}\left(N, T^{I}\right) & \stackrel{\left(1 \otimes p_{i *}\right)_{i}}{\longrightarrow} & \left(M \otimes_{R} \operatorname{Hom}(N, T)\right)^{I} \\
\Phi \downarrow & \downarrow \Phi^{I} \\
\operatorname{Hom}\left(\operatorname{Hom}_{R}(M, N), T^{I}\right) & \stackrel{\left(p_{i *}\right)_{i}}{\longrightarrow} & \operatorname{Hom}\left(\operatorname{Hom}_{R}(M, N), T\right)^{I}
\end{array}
$$

The injectivity of (3) follows, in view of the injectivity of $\Phi$ (the abelian group $\Delta=T^{I}$ being divisible) and the fact that the horizontal map at the bottom of the diagram is bijective.

Corollary 2.2. If $M$ is a right $R$-module which is strict Mittag-Leffler over the right regular module $R$, then $M$ is Mittag-Leffler with respect to the class $\mathfrak{I}$ of injective left $R$-modules. In other words, we have an inclusion $\operatorname{SML}(R) \subseteq \operatorname{ML}(\mathfrak{I})$.

Proof. Since the left $R$-module $D R=\operatorname{Hom}(R, \mathbb{Q} / \mathbb{Z})$ is an injective cogenerator of the category of left $R$-modules, it follows from Remark 1.1(iv) that $\operatorname{ML}(D R)=\operatorname{ML}(\mathfrak{I})$. Hence, the result is an immediate consequence of Proposition 2.1.

Let $N$ be a right $R$-module and consider a family $\left(M_{\lambda}\right)_{\lambda}$ of right $R$-modules and the direct sum $M=\bigoplus_{\lambda} M_{\lambda}$. Then, $M \in \operatorname{SML}(N)$ if and only if $M_{\lambda} \in \operatorname{SML}(N)$ for any $\lambda$. Indeed, it is easily seen that the additive map $\Phi_{M}$ of (2) is injective if and only if the corresponding maps $\Phi_{M_{\lambda}}$ are injective for all $\lambda$. Since the regular right $R$-module $R$ is obviously contained in $\operatorname{SML}(N)$, we conclude that $\operatorname{SML}(N)$ contains all projective right $R$-modules. Therefore, as with the case of Mittag-Leffler modules, it follows that if $K_{n}$ and $K_{n}^{\prime}$ are the $n$-th syzygy modules of a right $R$-module $M$ that correspond to two projective resolutions of it, then $K_{n}$ is strict Mittag-Leffler over $N$ if and only if this is the case for $K_{n}^{\prime}$. In that case, we say that the $n$-th syzygy module of $M$ is strict Mittag-Leffler over $N$.

The invariant silp $R$ of the ring $R$ was defined by Gedrich and Gruenberg in [9], as the supremum of the injective lengths of projective left $R$-modules. We denote by silp $R^{o p}$ the corresponding invariant which is defined using right $R$-modules. We recall that the ring $R$ is called right $\aleph_{0}$-Noetherian if all right ideals of it are countably generated and record the following result, which is an immediate consequence of [6, Theorem 3.1].

Theorem 2.3. Let $R$ be a right $\aleph_{0}$-Noetherian ring and assume that silp $R^{o p} \leq n<\infty$. Then, the $n$-th syzygy of any right $R$-module is strict Mittag-Leffler over the right $R$-module $R$.

Using Corollary 2.2, we obtain the following result.

Corollary 2.4. Let $R$ be a right $\aleph_{0}$-Noetherian ring and assume that silp $R^{o p} \leq n<\infty$. Then, the $n$-th syzygy of any right $R$-module is Mittag-Leffler with respect to the class $\mathfrak{I}$ of injective left $R$-modules.

\section{On the InJECTIVE LENGTH OF THE REgUlaR MODUle}

Let $R$ be a ring and consider the invariant silp $R$. Our goal in this section is to examine the extent to which the obvious inequality $\operatorname{id}_{R} R \leq \operatorname{silp} R$ is actually an equality. Since any projective left $R$-module is a direct summand of a suitable direct sum of copies of $R$, the problem consists in showing that the vanishing of the functor $\operatorname{Ext}_{R}^{n}(-, R)$ for some $n$ implies the vanishing of the functor $\operatorname{Ext}_{R}^{n}\left(-, R^{(\Lambda)}\right)$ for any set $\Lambda$.

Remarks 3.1. (i) If the ring $R$ is left Noetherian, then it is easily seen that $\operatorname{id}_{R} R=\operatorname{silp} R$. 
Indeed, assuming that $\operatorname{id}_{R} R=n<\infty$, we may construct an injective resolution of $R$ of length $n$. Since a direct sum of injective left $R$-modules is injective in this case (see [2, Theorem 1.1]), we may construct an injective resolution of $R^{(\Lambda)}$ of length $n$ for any set $\Lambda$.

(ii) Let $R$ be a left perfect and right coherent ring. Then, as shown in [14, Proposition 3], the injection $R^{(\Lambda)} \hookrightarrow R^{\Lambda}$ splits for any set $\Lambda$. It follows that any projective left $R$-module $P$ is a direct summand of the direct product $R^{\Lambda}$ for some set $\Lambda$. Since the direct product of injective left $R$-modules is injective, we conclude that $\operatorname{id}_{R} R^{\Lambda}=\mathrm{id}_{R} R$ for any set $\Lambda$ and hence $\operatorname{id}_{R} P \leq \operatorname{id}_{R} R$ for any projective left $R$-module. It follows readily that $\operatorname{silp} R=\mathrm{id}_{R} R$.

Lemma 3.2. Let $R$ be a countable ring and consider two countably generated left $R$-modules $M, N$, such that $\operatorname{Ext}_{R}^{1}(M, N)=0$. Then, $M$ is strict Mittag-Leffler over $N$.

Proof. We proceed as in the proof of [5, Proposition 1.2]. We write $M$ as the direct limit of a countable direct system $\left(M_{i}\right)_{i}$ of finitely presented left $R$-modules. Then, the Ext-groups of $M$ may be expressed in terms of the Ext-groups of the $M_{i}$ 's. In particular, there is a short exact sequence of abelian groups

$$
0 \longrightarrow \lim ^{1} \operatorname{Hom}_{R}\left(M_{i}, N\right) \longrightarrow \operatorname{Ext}_{R}^{1}(M, N) \longrightarrow \lim _{\leftarrow} \operatorname{Ext}_{R}^{1}\left(M_{i}, N\right) \longrightarrow 0
$$

and hence our assumption implies that $\lim _{\leftarrow i}^{1} \operatorname{Hom}_{R}\left(M_{i}, N\right)=0$. Since the left $R$-module $M_{i}$ is finitely generated, the abelian group $\operatorname{Hom}_{R}\left(M_{i}, N\right)$ is countable; indeed, if $M_{i}$ can be generated by $a_{i}$ elements as an $R$-module, then $\operatorname{Hom}_{R}\left(M_{i}, N\right)$ embeds into $N^{a_{i}}$. This being the case for all $i$, we may invoke Gray's criterion [10] in order to conclude that the triviality of the group $\lim ^{1}{ }_{i} \operatorname{Hom}_{R}\left(M_{i}, N\right)$ implies that the inverse system $\left(\operatorname{Hom}_{R}\left(M_{i}, N\right)\right)_{i}$ satisfies the Mittag-Leffler condition. Since the index set is countable, it follows that the latter inverse system satisfies the strict Mittag-Leffler condition, as needed.

Theorem 3.3. If $R$ is a countable ring, then $\operatorname{silp} R=i d_{R} R$.

Proof. Since we always have $\operatorname{id}_{R} R \leq \operatorname{silp} R$, it only remains to show that $\operatorname{silp} R \leq \operatorname{id}_{R} R$. Of course, we may assume that $\operatorname{id}_{R} R=n<\infty$. Since the countable ring $R$ is obviously left $\aleph_{0}$-Noetherian, the inequality silp $R \leq n$ will follow from [6, Theorem 3.1], provided that we show the $n$-th syzygy of any countably generated left $R$-module to be strict Mittag-Leffler over $R$. To that end, let $M$ be a countably generated left $R$-module and consider a resolution of it by countably generated free left $R$-modules. If $K=K_{n}$ is the corresponding $n$-th syzygy, then $\operatorname{Ext}_{R}^{1}(K, R)=\operatorname{Ext}_{R}^{n+1}(M, R)=0$. Invoking Lemma 3.2, we may conclude that $K$ is strict Mittag-Leffler over $R$.

Corollary 3.4. (cf. [13]) A countable left self-injective ring is quasi-Frobenius.

Proof. If a ring $R$ is countable and left self-injective, then Theorem 3.3 implies that silp $R=$ 0 . Invoking a result by Faith and Walker (cf. [7] and [8]), it follows that the ring $R$ is then quasi-Frobenius.

Remark 3.5. Let $R$ be a ring and consider a left $R$-module $A$ of finite projective dimension. Then, we always have $\operatorname{pd}_{R} A \leq \operatorname{silp} R$. (Indeed, if $\operatorname{pd}_{R} A=n<\infty$, there exists a projective left $R$-module $P$ with $\operatorname{Ext}_{R}^{n}(A, P) \neq 0$; in particular, it follows that $\operatorname{id}_{R} P \geq n$ and hence silp $R \geq n$.) If the $\operatorname{ring} R$ is countable, then, in view of Theorem 3.3 above, the latter inequality can be equivalently rewritten as $\operatorname{pd}_{R} A \leq \operatorname{id}_{R} R$ (cf. [6, Corollary 2.25]).

Since the integral group ring $\mathbb{Z} G$ of a countable group $G$ is countable, the following result is an immediate consequence of Theorem 3.3. 
Corollary 3.6. If $G$ is a countable group, then silp $\mathbb{Z} G=i d_{\mathbb{Z} G} \mathbb{Z} G$.

It is worth pointing out that Corollary 3.6 above complements [6, Theorem 4.8(iii)], where it is shown that $\operatorname{silp} \mathbb{Z} G=\operatorname{id}_{\mathbb{Z} G} \mathbb{Z} G$ for any countable group $G$, provided that $\operatorname{silp} \mathbb{Z} G<\infty$.

\section{ON THE RELATion Between SiLP $\mathbb{Z} G$ AND $\underline{\text { HD }} G$}

As shown in [5], for any group $G$ the invariant $\operatorname{silp} \mathbb{Z} G$ is equal to spli $\mathbb{Z} G$, i.e. to the supremum of the projective lengths of injective $\mathbb{Z} G$-modules. The finiteness of these invariants has an important geometric significance: If $G$ is a group with periodic cohomology after some steps, then these invariants are finite if and only if $G$ acts freely on a finite dimensional CWcomplex, which is homotopy equivalent to a sphere (cf. [16]). More generally, it is conjectured in [17] that the finiteness of these invariants is equivalent to the existence of a finite dimensional model for the classifying space for proper actions of $G$; this has been established for a big class of groups in [18].

Ikenaga defined in [12] the generalized cohomological dimension $\underline{\mathrm{cd}} G$ of a group $G$ as the supremum of the set of integers $n$, for which the abelian group $\operatorname{Ext}_{\mathbb{Z} G}^{n}(M, P)$ is non-trivial for some $\mathbb{Z}$-free $\mathbb{Z} G$-module $M$ and some projective $\mathbb{Z} G$-module $P$. Dembegioti and Talelli conjectured in [3, Conjecture A] that the invariants $\underline{\mathrm{cd}} G$ and spli $\mathbb{Z} G$ are related by the equality spli $\mathbb{Z} G=\underline{\mathrm{cd}} G+1$. They showed that this is indeed the case if $G$ is:

(i) a duality group or

(ii) the fundamental group of a graph of finite groups or

(iii) the fundamental group of the finite graphs of groups of type $F P_{\infty}$ that are described in [3, Theorem 3.5].

As shown in [5, Corollary 4.7(i)], we always have $\underline{\mathrm{cd}} G \leq \operatorname{spli} \mathbb{Z} G \leq \underline{\mathrm{cd}} G+1$. Therefore, the above conjecture is equivalent to the assertion that

$$
\underline{\mathrm{cd}} G+1 \leq \operatorname{spli} \mathbb{Z} G \text {. }
$$

We now consider the generalized homological dimension hd $G$ of $G$, which is defined as the supremum of the set of integers $n$, for which the abelian group $\operatorname{Tor}_{n}^{\mathbb{Z} G}(I, M)$ is non-trivial for some injective $\mathbb{Z} G$-module $I$ and some torsion-free $\mathbb{Z} G$-module $M$. Since hd $G \leq \underline{\mathrm{cd}} G$ (cf. [6, Theorem 4.11]), we may regard the validity of the inequality

$$
\underline{\text { hd }} G+1 \leq \operatorname{spli} \mathbb{Z} G
$$

as a weak form of the above conjecture. Let $\mathfrak{X}$ be the class consisting of those groups $G$ for which the inequality (5) is true. It is clear that $\mathfrak{X}$ contains all groups $G$, for which (4) is true; in particular, this is the case for the groups described in (i), (ii) and (iii) above. If $G$ is a locally finite group, then $\underline{\mathrm{hd}} G=0$ (cf. [12, §III, Corollary 2]) and hence $G \in \mathfrak{X}$.

Theorem 4.1. Let $N$ be a normal subgroup of a group $G$. If $N \in \mathfrak{X}$ and the quotient group $\bar{G}=G / N$ is locally finite, then $G \in \mathfrak{X}$.

Proof. In order to show that $G \in \mathfrak{X}$, we may assume that spli $\mathbb{Z} G=n<\infty$. We note that $N \in \mathfrak{X}$ is a subgroup of $G$ and hence

$$
\underline{\text { hd }} N+1 \leq \operatorname{spli} \mathbb{Z} N \leq \operatorname{spli} \mathbb{Z} G=n
$$

(cf. $[9,5.1$ (iii)]), i.e. hd $N \leq n-1$. We have to show that $\underline{\text { hd }} G+1 \leq n$, i.e. that hd $G \leq n-1$. Since $G$ is the directed union of its countable subgroups, we may use the behavior of hd under directed unions (cf. [12, §III, Proposition 9]) and conclude that it suffices to prove that $\underline{\text { hd }} G_{0} \leq n-1$ for any countable subgroup $G_{0} \subseteq G$. To that end, let us consider a countable 
subgroup $G_{0} \subseteq G$. We note that $G_{0}$ is an extension of a subgroup $\bar{G}_{0}$ of $\bar{G}$ by a subgroup $N_{0}$ of $N$. The group $\bar{G}_{0}$ is, of course, locally finite and we have inequalities spli $\mathbb{Z} G_{0} \leq$ spli $\mathbb{Z} G=n$ (cf. [9, 5.1(iii)]) and hd $N_{0} \leq \underline{\text { hd }} N \leq n-1$ (cf. [12, §III, Proposition 8]). Then, the inequality hd $G_{0} \leq n-1$ follows from the following result.

Proposition 4.2. Let $N$ be a normal subgroup of a countable group $G$, such that the quotient group $\bar{G}=G / N$ is locally finite. Assume that $n$ is an integer, such that spli $\mathbb{Z} G \leq n$ and $\underline{h d} N \leq n-1$. Then, $\underline{h d} G \leq n-1$.

Proof. We consider two $\mathbb{Z} G$-modules $I$ and $M$, with $I$ injective and $M$ torsion-free, and the diagonal $\mathbb{Z} G$-module $I \otimes M$. The groups $\operatorname{Tor}_{*}^{\mathbb{Z} G}(I, M)=H_{*}(G, I \otimes M)$ can be computed by means of the Lyndon-Hochshild-Serre spectral sequence

$$
E_{p q}^{2}=H_{p}\left(\bar{G}, H_{q}(N, I \otimes M)\right) \Longrightarrow H_{p+q}(G, I \otimes M) .
$$

We are interested in the filtration of the homology group $H_{n}(G, I \otimes M)$, which is induced by that spectral sequence. Since the $\mathbb{Z} N$-module $\operatorname{res}_{N}^{G} I$ is injective, whereas $\operatorname{res}_{N}^{G} M$ is torsionfree, our assumption that hd $N \leq n-1$ implies that $H_{n}(N, I \otimes M)=\operatorname{Tor}_{n}^{\mathbb{Z N}}(I, M)=0$. In particular, $E_{0 n}^{2}=H_{0}\left(\bar{G}, H_{n}(N, I \otimes M)\right)=0$. On the other hand, since $\bar{G}$ is locally finite, the abelian group $E_{p q}^{2}=H_{p}\left(\bar{G}, H_{q}(N, I \otimes M)\right)$ is torsion for all $p>0$. Being a subquotient of the latter, the limit term $E_{p q}^{\infty}$ of the spectral sequence is torsion for all $p>0$ as well. We therefore conclude that the group $H_{n}(G, I \otimes M)$ admits a finite filtration all of whose quotients are torsion groups. Hence, $\operatorname{Tor}_{n}^{\mathbb{Z} G}(I, M)=H_{n}(G, I \otimes M)$ is itself a torsion group. Since the ring $\mathbb{Z} G$ is countable and silp $\mathbb{Z} G=$ spli $\mathbb{Z} G \leq n$ (cf. [5, Corollary 4.5]), we may invoke the following lemma and conclude that $\operatorname{Tor}_{n}^{\mathbb{Z} G}(I, M)=0$. This being the case for any two $\mathbb{Z} G$-modules $I$ and $M$, with $I$ injective and $M$ torsion-free, it follows that $\underline{\mathrm{hd}} G \leq n-1$.

Lemma 4.3. Let $R$ be a left $\aleph_{0}$-Noetherian ring, i.e. a ring all of whose left ideals are countably generated. Assume that $n$ is an integer, such that silp $R \leq n$, and consider a left $R$-module $M$, which is torsion-free as an abelian group. Then:

(i) (cf. [3, Lemma 2.1]) the abelian group $\operatorname{Ext}_{R}^{n}(M, R)$ is divisible and

(ii) the abelian group $\operatorname{Tor}_{n}^{R}(I, M)$ is torsion-free for any injective right $R$-module $I$.

Proof. (i) Let $t$ be a positive integer and consider the injective $R$-linear map $f: M \longrightarrow M$,

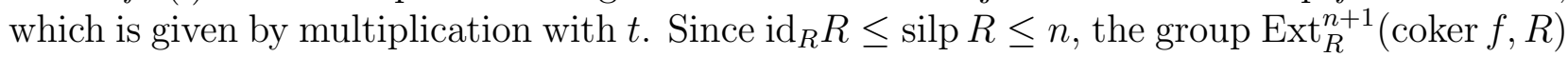
is trivial and hence the induced map $f^{*}: \operatorname{Ext}_{R}^{n}(M, R) \longrightarrow \operatorname{Ext}_{R}^{n}(M, R)$ is surjective. The latter map being multiplication with $t$, it follows that the group $\operatorname{Ext}_{R}^{n}(M, R)$ is $t$-divisible. This is the case for all $t \geq 1$ and hence the group $\operatorname{Ext}_{R}^{n}(M, R)$ is divisible.

(ii) Since any injective right $R$-module is a direct summand of a cofree module, we can easily reduce to the case where $I=\operatorname{Hom}(R, \Delta)$ for some divisible abelian group $\Delta$. Then, as shown in [6, Theorem 3.1 and Proposition 1.5], our assumption that silp $R \leq n$ implies that the group $\operatorname{Tor}_{n}^{R}(I, M)=\operatorname{Tor}_{n}^{R}(\operatorname{Hom}(R, \Delta), M)$ may be embedded into the group $\operatorname{Hom}\left(\operatorname{Ext}_{R}^{n}(M, R), \Delta\right)$. Therefore, it suffices to show that the abelian group $\operatorname{Hom}\left(\operatorname{Ext}_{R}^{n}(M, R), \Delta\right)$ is torsion-free. This follows from (i) above, in view of the following elementary observation: If $A, B$ are two abelian groups and $A$ is divisible, then the abelian group $\operatorname{Hom}(A, B)$ is torsion-free.

\section{ON The flat Length OF $D R$}

Let $R$ be a ring and consider the invariant sfli $R$, which is defined as the supremum of the flat lengths of injective left $R$-modules. This invariant was introduced in [4] and has been also studied in $[6]$. Since $D R=\operatorname{Hom}(R, \mathbb{Q} / \mathbb{Z})$ is an injective left $R$-module, it is clear that 
$\mathrm{fd}_{R} D R \leq \operatorname{sfli} R$. Our goal in this section is to examine the extent to which the latter inequality is actually an equality. Since any injective left $R$-module is a direct summand of a suitable direct product of copies of $D R$, the problem consists in showing that the vanishing of the functor $\operatorname{Tor}_{n}^{R}\left({ }_{-}, D R\right)$ for some $n$ implies the vanishing of the functor $\operatorname{Tor}_{n}^{R}\left({ }_{-}, D R^{\Lambda}\right)$ for any set $\Lambda$. More generally, we shall examine the question above for any injective cogenerator $J$ of the category of left $R$-modules.

Proposition 5.1. Let $R$ be a ring and consider an injective cogenerator $J$ of the category of left $R$-modules. Then, the following conditions are equivalent for a non-negative integer $n$ :

(i) sfli $R \leq n$ and

(ii) $f d_{R} J \leq n$ and the $(n+1)$-th syzygy of any right $R$-module is Mittag-Leffler with respect to the class $\mathfrak{J}$ of injective left $R$-modules.

Proof. (i) $\rightarrow$ (ii): Assuming that sfli $R \leq n$, the inequality $\mathrm{fd}_{R} J \leq$ sfli $R$ shows that we also have $\operatorname{fd}_{R} J \leq n$. On the other hand, since the class $\mathfrak{J}$ of injective left $R$-modules is closed under direct products and the functor $\operatorname{Tor}_{n+1}(-, I)$ vanishes for any injective left $R$-module $I$ (as $\mathrm{fd}_{R} I \leq$ sfli $R \leq n$ ), we may invoke Proposition 1.2 and conclude that the $(n+1)$-th syzygy of any right $R$-module is Mittag-Leffler with respect to $\mathfrak{J}$.

(ii) $\rightarrow$ (i): Since any injective left $R$-module is a direct summand of a suitable direct product of copies of the injective cogenerator $J$, it suffices to show that $\mathrm{fd}_{R} J^{\Lambda} \leq n$ for any set $\Lambda$. To that end, we fix a set $\Lambda$ and note that our assumption on the syzygies of right $R$-modules implies, in view of Proposition 1.2, that the natural map

$$
\varphi_{M}^{(n+1)}: \operatorname{Tor}_{n+1}^{R}\left(M, J^{\Lambda}\right) \longrightarrow \operatorname{Tor}_{n+1}^{R}(M, J)^{\Lambda}
$$

is injective for any right $R$-module $M$. Since $\operatorname{fd}_{R} J \leq n$, the group $\operatorname{Tor}_{n+1}^{R}(M, J)$ is trivial and hence we may conclude that $\operatorname{Tor}_{n+1}^{R}\left(M, J^{\Lambda}\right)=0$, as needed.

Corollary 5.2. If $R$ is a right $\aleph_{0}$-Noetherian ring and $J$ an injective cogenerator of the category of left $R$-modules, then sfli $R \leq \max \left\{f d_{R} J\right.$, silp $\left.R^{o p}-1\right\}$.

Proof. The inequality to be proved is obvious if $\max \left\{\mathrm{fd}_{R} J\right.$, silp $\left.R^{o p}-1\right\}=\infty$ and hence we may assume that $\max \left\{\operatorname{fd}_{R} J, \operatorname{silp} R^{o p}-1\right\}=n<\infty$. Then, we have silp $R^{o p} \leq n+1$ and hence Corollary 2.4 implies that the $(n+1)$-th syzygy of any right $R$-module is Mittag-Leffler with respect to the class $\mathfrak{I}$ of injective left $R$-modules. Since we also have $\mathrm{fd}_{R} J \leq n$, the inequality sfli $R \leq n$ follows from Proposition 5.1.

We note that if $R$ is a right $\aleph_{0}$-Noetherian ring, then we have an inequality sfli $R \leq \operatorname{silp} R^{o p}$ (cf. [5, Proposition 3.2]).

Corollary 5.3. Let $R$ be a right $\aleph_{0}$-Noetherian ring and assume that one of the following two conditions is satisfied:

(i) sfli $R=\operatorname{silp} R^{o p}<\infty$ or

(ii) $\operatorname{silp} R^{o p} \leq 1$.

Then, $f d_{R} J=\overline{s f l i} R$ for any injective cogenerator $J$ of the category of left $R$-modules.

Proof. Let $J$ be an injective cogenerator of the category of left $R$-modules.

(i) If $\mathrm{fd}_{R} J<\operatorname{sfli} R$, then Corollary 5.2 implies that sfli $R \leq \operatorname{silp} R^{o p}-1$, contradicting our hypothesis that sfli $R=\operatorname{silp} R^{o p}<\infty$.

(ii) In view of (i) above, it only remains to consider the case where the inequality sfli $R \leq$ silp $R^{o p}$ of [5, Proposition 3.2] is strict. In that case, our assumption implies that sfli $R=0$; hence, the obvious inequality $\operatorname{fd}_{R} J \leq \operatorname{sfli} R$ is necessarily an equality. 
Remark 5.4. The class of right $\aleph_{0}$-Noetherian rings $R$ for which silp $R^{o p} \leq 1$ includes the quasi-Frobenius rings and the integral group rings of finite groups. Indeed, as shown by Faith and Walker in [7] and [8], the quasi-Frobenius rings are precisely the rings $R$ for which silp $R^{o p}=0$. On the other hand, Gedrich and Gruenberg have shown in $[9, \S 5.2]$ that $\operatorname{silp} \mathbb{Z} G=1$ for any finite group $G$.

Corollary 5.5. Let $R$ be a ring and assume that one of the following conditions is satisfied:

(i) $R$ is right Noetherian,

(ii) $R$ is right $\aleph_{0}$-Noetherian, right perfect and left coherent or

(iii) $R$ is countable.

Then, for any injective cogenerator $J$ of the category of left $R$-modules, we have an inequality sfli $R \leq \max \left\{f d_{R} J, i d_{R^{o p}} R-1\right\}$. Moreover, if we also have sfli $R=i d_{R^{o p}} R<\infty$ or $i d_{R^{o p}} R \leq 1$, then sfli $R=f d_{R} J$.

Proof. Our assumptions on $R$ imply that $\operatorname{silp} R^{o p}=\operatorname{id}_{R^{o p}} R$ (cf. Remarks 3.1 and Theorem 3.3). Therefore, the result is simply a restatement of Corollaries 5.2 and 5.3 .

Acknowledgements. This paper is part of the author's $\mathrm{PhD}$ thesis at the University of Athens. I would like to thank my advisor Ioannis Emmanouil for his help and suggestions during its preparation. I would also like to thank Apostolos Beligiannis for some useful remarks.

\section{REFERENCES}

[1] Angeleri-Hügel, L., Herbera, D.: Mittag-Leffler conditions on modules. Ind. Univ. Math. J. 57, 2459-2517 (2008)

[2] Bass, H.: lnjective dimension in Noetherian rings. Trans. Am. Math. Soc. 102, 18-29 (1962).

[3] Dembegioti, F., Talelli, O.: On a relation between certain cohomological invariants. J. Pure Appl. Algebra 212, 1432-1437 (2008)

[4] Ding, N., Chen, J.: The flat dimensions of injective modules. Manuscripta Math. 78, $165-177$ (1993)

[5] Emmanouil, I.: On certain cohomological invariants of groups. Adv. Math. 225, 3446-3462 (2010)

[6] Emmanouil, I., Talelli, O.: On the flat length of injective modules. J. London Math. Soc. 84, 408-432 (2011)

[7] Faith, C.: Rings with ascending condition on annihilators. Nagoya Math. J. 27, 179-191 (1966)

[8] Faith, C., Walker, E.A.: Direct-sum representations of injective modules. J. Algebra 5, 203-221 (1967)

[9] Gedrich, T.V., Gruenberg, K.W.: Complete cohomological functors on groups. Topology Appl. 25, 203$223(1987)$

[10] Gray, B.: Spaces of the same $n$-type, for all $n$. Topology 5, 241-243 (1966)

[11] Grothendieck, A.: EGA III. Publ. Math. IHES 11 (1961)

[12] Ikenaga, B.M.: Homological dimension and Farrell cohomology. J. Algebra 87, 422-457 (1984)

[13] Lawrence, J.: A countable self-injective ring is quasi-Frobenius. Proc. Amer. Math. Soc. 65 217-220 (1977)

[14] Lenzing, H.: Direct sums of projective modules as direct summands of their direct product. Comm. Algebra 4, 681-691 (1976)

[15] Raynaud, M., Gruson, L.: Critères de platitude et de projectivité. Invent. Math. 13, 1-89 (1971)

[16] Talelli, O.: Periodicity in group cohomology and complete resolutions. Bull. London Math. Soc. 37, $547-554$ (2005)

[17] Talelli, O.: On groups of type $\Phi$. Archiv der Mathematik 89, 24-32 (2007)

[18] Talelli, O.: A characterization of cohomological dimension for a big class of groups. J. Algebra 326, 238-244 (2011)

Department of Mathematics, University of Athens, Athens 15784, Greece

E-mail address: dballas@math.uoa.gr 